\title{
A METHOD FOR THE SOLUTION OF THE DIRICHLET PROBLEM FOR CERTAIN TYPES OF DOMAINS*
}

\author{
BY \\ BERNARD EPSTEIN \\ University of Pennsylvania
}

1. Introduction. It is our purpose to present a method for solving the first boundaryvalue problem of potential theory - frequently called the Dirichlet problem-for a class of domains which may be considered as limiting cases of those domains for which the so-called "alternating procedure" of H. A. Schwarz [2, p. 264] is applicable. As is well known, the Schwarz method enables us to solve the Dirichlet problem for a domain which is the sum of two overlapping domains, for each of which it is known how to solve this kind of problem. In this paper we consider the case where the given domain is the sum of two domains which are adjacent, rather than overlapping, and for each of which it is known how to solve the Dirichlet problem.

As will be explained in detail in Sec. 2, the underlying idea is that the problem would be essentially solved if the values of the desired function could be determined at the points common to the two component domains, for the complete solution could then be found by solving Dirichlet problems for each of the component domains. Assuming that the boundaries consist of sufficiently smooth arcs, we derive an integral equation which governs the values of the solution on the boundary arcs common to the two domains. A method of successive approximation is presented for the solution of this integral equation, so that the original problem is essentially solved.

The connection between the Schwarz procedure and that which is presented here becomes particularly clear if we consider R. Nevanlinna's approach to the former [4]. He shows that the original problem of determining a certain harmonic function of two variables can be reduced, whenever the Schwarz procedure is applicable, to the problem of solving an integral equation for a function of one variable, and then solves the integral equation by a method similar to that described in this paper. However, it should be emphasized that the method by which the integral equation considered here is derived, is quite different from that employed by Nevanlinna, and that these two integral equations arise in two entirely different problems, the one considered here being a limiting case of that considered by Schwarz and Nevanlinna.

2. Statement of the problem and derivation of the integral equation. We consider a plane domain $S$ of finite connectivity, whose boundary is composed of a finite number of smooth arcs. We suppose that, by the addition of a finite number of smooth arcs to the boundary, the domain $S$ is divided into two adjacent domains whose Green's functions are known. For the sake of definiteness we shall consider the domain shown in Fig. 1, but it will be evident how our method can be applied to other domains.

*Received Nov. 3, 1947. This paper is a condensed version of a thesis submitted in partial fulfillment of the requirements for the degree of Doctor of Philosophy at Brown University, June, 1947.

The Schwarz method can be immediately extended to domains consisting of three or more overlapping domains [2, p. 264]. Similarly, the method described in this paper can be generalized to the case of more than two component domains. Also, we shall consider only the two-dimensional case; the extension to three or more dimensions is quite evident, as in the case of the Schwarz procedure. 
Referring to Fig. 1, we assume that the functions ${ }^{2} h_{1}(s), h_{2}(s), h_{3}(s), h_{4}(s)$ are prescribed on $A B C, A D C$, and the upper and lower edges of $E F$, respectively. Moreover, we assume that each of these functions is bounded and possesses at most a finite number of discontinuities. We are then assured [3, p. 22] of the existence of a unique solution to the Dirichlet problem, i.e., a function $u(P), P=(x, y)$, which is bounded and har-

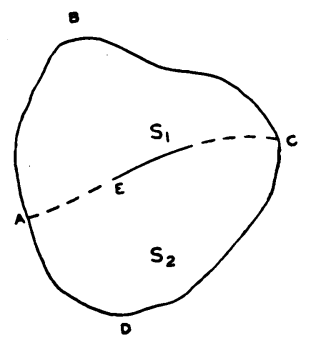

Fig. 1.

monic in the interior of $S$ and which approaches the prescribed boundary values at every point of continuity.

Now suppose that by the addition of the smooth (or piecewise smooth) arcs $A E$ and $F C$ the original domain $S$ is divided into two domains $S_{1}$ and $S_{2}$ whose Green's functions, $G_{1}$ and $G_{2}$ respectively, are known. On these added ares the function $u(P)$ becomes a function $f(s)$ of the arc-length. It is clear that the problem of determining $u(P)$ would be essentially solved if the function $f(s)$ could be found, for the value of $u(P)$ at any point of $S$ not lying on the added arcs could then be expressed in terms of the boundary-values, the function $f(s)$, and the Green's functions $G_{1}$ and $G_{2}$. Indeed, the value of $u$ at any interior point $P$ of $S_{1}$ could be expressed as follows:

$$
u(P)=\frac{1}{2 \pi}\left\{\int_{\Delta B C} h_{1}(s) \frac{\partial G_{1}}{\partial n} d s+\int_{E F} h_{3}(s) \frac{\partial G_{1}}{\partial n} d s+\int_{A E+F C} f(s) \frac{\partial G_{1}}{\partial n} d s\right\},
$$

where $\partial / \partial n$ indicates differentiation in the direction of the inward normal and the Green's function $G_{1}$ is evaluated at the pair of points $(P, Q), Q$ describing the boundary of $S_{1}$. Similarly, when the point $P$ is taken in $S_{2}$ the value of $u(P)$ is given by

$$
u(P)=\frac{1}{2 \pi}\left\{\int_{A D C} h_{2}(s) \frac{\partial G_{2}}{\partial n} d s+\int_{E F} h_{4}(s) \frac{\partial G_{2}}{\partial n} d s+\int_{A E+F C} f(s) \frac{\partial G_{2}}{\partial n} d s\right\} .
$$

The first two integrals appearing in each of the two above equations define a certain function throughout the interiors of $S_{1}$ and $S_{2}$. If this function is designated in both domains as $w(P)$, then Eqs. (2.1) and (2.2) can be written as a single equation:

$$
u(P)=w(P)+\frac{1}{2 \pi} \int_{A E+P C} f(s) \frac{\partial G}{\partial n} d s,
$$

where $G$ is taken as $G_{1}$ or $G_{2}$, depending on whether the point $P$ lies in $S_{1}$ or $S_{2}$.

Now let $P$ be any inner point, temporarily fixed, of arc $A E$ or $F C$, and let $R$ be a positive number such that the interior of $C_{R}(P)^{3}$ lies entirely inside $S$ and its circum-

2The variable $s$ always measures length along the arc under consideration.

${ }^{3} C_{R}(P)$ is used to indicate the circle with the center $P$ and the radius $R$. 
ference meets the boundaries of $S_{1}$ and $S_{2}$ (including the added arcs) at only a finite number of points. (For example, if part of arc $A B C$ coincides with part of the circumference of $C_{R^{\prime}}(P)$, it is necessary to choose $R<R^{\prime}$.) Upon exclusion of these points the circumference of $C_{R}(P)$ divides into a finite number of open ares each of which lies entirely inside $S_{1}$ or entirely inside $S_{2}$. Now let the point $Q$ describe the $\operatorname{arcs} A E$ and $F C$, let $P^{\prime}$ describe the circumference of $C_{R}(P)$, and let the point $P$ correspond to $s=s_{0}$. Then the mean-value property for the harmonic function $u$ at the point $P$ may be expressed as follows:

$$
\begin{aligned}
f\left(s_{0}\right) & =\frac{1}{2 \pi} \int_{0}^{2 \pi} u\left(P^{\prime}\right) d \theta \\
& =\frac{1}{2 \pi} \int_{0}^{2 \pi} w\left(P^{\prime}\right) d \theta+\frac{1}{4 \pi^{2}} \int_{0}^{2 \pi}\left\{\int_{A E+F C} f\left(s_{\theta}\right) \frac{\partial G\left(P^{\prime}, Q\right)}{\partial n_{Q}} d s_{\theta}\right\} d \theta,
\end{aligned}
$$

where $\theta$ is the angle between $P P^{\prime}$ and some fixed direction, say the positive direction of the $x$-axis, and $G$ is taken as $G_{1}$ or $G_{2}$, as explained following (2.3).

Now, by Fubini's theorem [5, p. 77], it is legitimate to invert the order of the iterated integral appearing in (2.4), so that the latter equation may be rewritten as follows:

$$
f\left(s_{0}\right)=\frac{1}{2 \pi} \int_{0}^{2 \pi} w\left(P^{\prime}\right) d \theta+\int_{A E+F C} f\left(s_{\odot}\right)\left\{\frac{1}{4 \pi^{2}} \int_{0}^{2 \pi} \frac{\partial G\left(P^{\prime}, Q\right)}{\partial n_{\odot}} d \theta\right\} d s_{\odot} .
$$

If there is now chosen for each point $P$ interior to the arcs $A E$ and $F C$ a quantity $R\left(s_{0}\right)$ subject only to the restriction mentioned above, the first term on the righthand side of (2.5) defines a certain function of $P$, henceforth designated $g_{R}\left(s_{0}\right)$, while the inner integral of the second term defines a function $K_{R}\left(s_{0}, s_{\diamond}\right)$ for every pair of inner points $P$ and $Q$ of the $\operatorname{arcs} A E$ and $F C$. (The subscript $R$ serves as a reminder that the functions $g$ and $K$ depend on the choice of the radius $R\left(s_{0}\right)$ over which the averaging is performed.) Thus, (2.5) becomes an integral equation of the second kind for the values of $u(P)$, the solution of the Dirichlet problem, along the added arcs. If $s_{0}$ is replaced by $s$ and $s_{0}$ by $s^{\prime}$, Eq. (2.5) becomes

$$
f(s)=g_{R}(s)+\int_{A E+F C} f\left(s^{\prime}\right) K_{R}\left(s, s^{\prime}\right) d s^{\prime} .
$$

The solution of the original Dirichlet problem has now been reduced to the solution of the integral equation (2.6).

Before we discuss the problem of solving (2.6), it would appear desirable to sketch briefly the nature of the kernel $K_{R}\left(s, s^{\prime}\right)$. As can be seen from (2.5), the kernel

$$
K_{R}\left(s, s^{\prime}\right)=\frac{1}{4 \pi^{2}} \int_{0}^{2 \pi} \frac{\partial G\left(P^{\prime}, Q\right)}{\partial n_{\odot}} d \theta
$$

is equal to the mean value over the circumference of the circle $C_{R}(P)$ (where $P$ corresponds to the parametric value $s$ along the added arcs) of $1 / 2 \pi$ times the inward normal derivative, at the point $Q$ corresponding to the parametric value $s^{\prime}$ along the added arcs, of the Green's function of the domain, $S_{1}$ or $S_{2}$, within which the point $P^{\prime}$, which describes the circumference of $C_{R}(P)$, and which is to be taken as the pole of the Green's function, lies. If we take account of the relationship existing between the Green's function and the harmonic measure [3, p. 30], and again use Fubini's theorem 
to justify the inversion of the order of integration, we find that the kernel function admits the following simple interpretation: The integral $\int K_{R}\left(s, s^{\prime}\right) d s^{\prime}$ taken over any portion of the added ares is equal to the mean value over the circumference of the circle $C_{R}(P)$ of the harmonic measure, evaluated at the point $P^{\prime}$ which describes the circumference, of that portion of the added ares, the measure being taken with respect to the domain $S_{1}$ or $S_{2}$ within which $P^{\prime}$ lies.

We shall show in Sec. 3 that the integral equation (2.6) can be solved by a method of successive approximation, provided that the kernel satisfies a certain condition. However, it is of interest to note that it is possible to obtain from (2.6) lower and upper bounds on the solution $f(s)$, quite independently of whether the method of solution which we shall describe in Sec. 3 is or is not applicable. Let $f_{0}(s)$ be any function which is known, from any considerations, ${ }^{4}$ to lie below the solution $f(s)$. If $f\left(s^{\prime}\right)$ is replaced on the right-hand side of $(2.6)$ by $f_{0}\left(s^{\prime}\right)$, it follows from the non-negative character of $K_{R}\left(s, s^{\prime}\right)$ that the resulting value of the right-hand side, which we shall designate $f_{1}(s)$, also lies below $f(s)$. If $f_{1}(s)$ is somewhere or everywhere larger than $f_{0}(s)$, then the function $\max \left(f_{0}(s), f_{1}(s)\right)$ will constitute an improved lower bound for $f(s)$. Similarly, it is possible to obtain improvements in upper bounds. Cf. Theorem 4.3.

3. Solution of the integral equation (2.6). It is clear that the integral equation (2.6) is, for fixed $s$, singular for two or more values of $s^{\prime}$, namely for those values of $s^{\prime}$ corresponding to the points at which the circumference of $C_{R}(P)$ meets the $\operatorname{arcs} A E$ and $F C$. Therefore, the Fredholm theory of linear integral equations [1, Chap. 3] is not applicable. However, the existence of at least one solution to (2.6) is assured by the existence theory for the Dirichlet problem.

We recall that the normal derivative of the Green's function (taken inward to the domain) is always positive, except perhaps at certain exceptional points, and, for any interior point $Q$ of the domain, that

$$
\frac{1}{2 \pi} \int \frac{\partial G(P, Q)}{\partial n_{P}} d s_{P}=1,
$$

the integration being taken around the entire boundary of the domain. Thus,

$$
\begin{aligned}
0<\int_{A E+F C} K_{R}\left(s, s^{\prime}\right) d s^{\prime} & =\frac{1}{4 \pi^{2}} \int_{A E+F C}\left\{\int_{0}^{2 \pi} \frac{\partial G\left(Q, P^{\prime}\right)}{\partial n_{P^{\prime}}} d \theta\right\} d s^{\prime} \\
& =\frac{1}{4 \pi^{2}} \int_{0}^{2 \pi}\left\{\int_{A E+F C} \frac{\partial G\left(Q, P^{\prime}\right)}{\partial n_{P^{\prime}}} d s^{\prime}\right\} d \dot{\theta}<\frac{1}{2 \pi} \int_{0}^{2 \pi} d \theta=1 .
\end{aligned}
$$

Now suppose that the function $R(s)$ can be so chosen that (3.2) can be replaced by the stronger inequality

$$
0<\int_{A E+F C} K_{R}\left(s, s^{\prime}\right) d s^{\prime} \leq p<1
$$

for all points $P$ interior to the $\operatorname{arcs} A E$ and $F C$, where $p$ is a certain positive constant. We have not been able to prove the possibility of so choosing $R(s)$ in the general case, but in Sec. 8 we shall show that this can be done for the class of domains considered in Sec. 6 , and it will be shown in Sec. 5 that this can also be done if the domain and

${ }^{4}$ For instance, $f_{0}(s)$ might be taken identically equal to the minimum of the boundary values. 
the added arcs satisfy certain conditions. In the present section and in Sec. 4 it is assumed that $R(s)$ has been so chosen that (3.3) is satisfied.

The importance of the condition (3.3) is two-fold. First, as we shall show immediately, it guarantees the uniqueness of a bounded ${ }^{5}$ solution of (2.6). Therefore, if we succeed in obtaining one solution, we can be certain that it is the function which provides the solution to the Dirichlet problem, and not some other function which, while it satisfies (2.6), may be entirely unrelated to the original problem from which (2.6) was derived. Secondly, the condition (3.3) makes it possible to solve equation (2.6) by a method which will be described in the present section.

We now prove that (3.3) guarantees the uniqueness of the bounded solution of (2.6). If there were two bounded solutions, $f(s)$ and $F(s)$, their difference, which would also be bounded, would satisfy the homogeneous equation

$$
F(s)-f(s)=\int_{A E+F C}\left(F\left(s^{\prime}\right)-f\left(s^{\prime}\right)\right) K_{R}\left(s, s^{\prime}\right) d s^{\prime} .
$$

From the inequality (3.3) there would follow

$$
\max |F(s)-f(s)| \leq p \max |F(s)-f(s)|,
$$

from which the identity of $f(s)$ and $F(s)$ is evident.

The unique bounded solution of (2.6) can be obtained by a method of successive approximation, as shown by the following theorem.

Theorem 3.1. Let $f_{0}(s)$ be any bounded continuous ${ }^{6}$ function defined on the arcs $A E$ and $F C$, and let the sequence of functions $\left\{f_{n}(s)\right\}, n=1,2,3, \cdots$ be defined by the recursion formula

$$
f_{n}(s)=g_{R}(s)+\int_{A E+F C} f_{n-1}\left(s^{\prime}\right) K_{R}\left(s, s^{\prime}\right) d s^{\prime} .
$$

(The function $R(s)$ is assumed continuous, or at least such that (3.6) has meaning for all $n$ and all s.) Then the sequence $\left\{f_{n}(s)\right\}$ converges uniformly to the unique bounded solution $f(s)$ of $(2.6)$.

Proof. Let the functions $\eta_{n}(s)$ be defined by the equations

$$
\eta_{n}(s)=f_{n}(s)-f(s), \quad n=0,1,2,3, \cdots .
$$

Then (3.6) may be written as follows:

$$
\eta_{n}(s)+f(s)=g_{R}(s)+\int_{A E+F C} \eta_{n-1}\left(s^{\prime}\right) K_{R}\left(s, s^{\prime}\right) d s^{\prime}+\int_{A E+F C} f\left(s^{\prime}\right) K_{R}\left(s, s^{\prime}\right) d s^{\prime} .
$$

Since $f(s)$ satisfies (2.6), (3.8) simplifies to

$$
\eta_{n}(s)=\int_{A E+F C} \eta_{n-1}\left(s^{\prime}\right) K_{R}\left(s, s^{\prime}\right) d s^{\prime}
$$

${ }^{5} \mathrm{As}$ demonstrated in [4] by means of an illustrative example, it is entirely conceivable that an integral equation such as (2.6) may possess an unbounded solution in addition to its unique bounded solution.

${ }^{6}$ It would suffice to require that $f_{0}(s)$ be bounded and integrable, but it seems unlikely that it would ever be practicable to choose for $f_{0}$ a discontinuous function. 
Designating ${ }^{7} \max \left|\eta_{n}(s)\right|$ by $M_{n}$, one obtains from (3.9)

$$
M_{n} \leq p M_{n-1},
$$

and, by repeated application of (3.10),

$$
M_{n} \leq p^{n} M_{0} .
$$

Thus, no matter how poor the original estimate $f_{0}(s)$ may be, ${ }^{8}$ the sequence of functions $\left\{\eta_{n}(s)\right\}$ converges uniformly to zero, and therefore the sequence $\left\{f_{n}(s)\right\}$ converges uniformly to $f(s)$. This completes the proof of Theorem 3.1.

Although it is possible according to Theorem 3.1 to choose the initial approximation $f_{0}(s)$ with almost complete arbitrariness, it might be expected that it is desirable to choose $f_{0}(s)$ in such a manner that it joins continuously with the prescribed boundary values, whenever such a choice is possible. A certain justification for this view is found in the following theorem, which we state without proof.

Theorem 3.2. Let the prescribed boundary values and the initial approximation $f_{0}(s)$ (which is assumed bounded) join continuously at any one of the end-points of the added arcs. Then the next approximation $f_{1}(s)$ also joins continuously with the boundary values at that point.

It is important to note that the procedure described in Theorem 3.1 is by no means restricted to the integral equation (2.6). It is easily seen that this procedure is applicable to any integral equation:

$$
f(s)=g(s)+\int_{a}^{b} K\left(s, s^{\prime}\right) f\left(s^{\prime}\right) d s^{\prime}
$$

for which the following conditions are satisfied ${ }^{9}$ :

(1) the equation possesses a (bounded) solution, and

(2) $\int_{a}^{b}\left|K\left(s, s^{\prime}\right)\right| d s^{\prime} \leq p, a \leq s \leq b$, where $p$ is any constant $<1$. It will be seen that Theorems 4.1 and 4.2 are also applicable, while, if the kernel is non-negative, Theorem 4.3 also holds.

4. Estimation of accuracy of approximation. In Sec. 3 a procedure was presented which in principle yields the solution of the integral equation (2.6), and therefore provides the solution of the Dirichlet problem from which (2.6) was derived. Equation (3.11) provides an upper bound for the maximum error $M_{n}$ at the n'th stage of the approximation procedure in terms of the maximum error $M_{0}$ of the initial estimate $f_{0}$. The principle of maximum and minimum for harmonic functions shows that the solution of (2.6) must lie between the maximum and the minimum of the prescribed boundary data. Thus, for example, if these bounds are $a$ and $b$ respectively, and if the initial estimate is chosen to be $(a+b) / 2$, then $M_{0}$, the least upper bound for $\left|\eta_{0}\right|$, does certainly not exceed $(a-b) / 2$, so that by (3.11) one immediately obtains for all further stages of the approximation procedure the estimate

TThe term "max" is to be understood as signifying the least upper bound. That all bounds are finite follows from the boundedness of the solution and of $f_{0}$.

8This means, no matter how large $M_{0}$ may be. An upper bound on $M_{0}$ can always be obtained from the fact that $f(s)$ must lie between the minimum and the maximum of the prescribed boundary data.

'If $g(s)$ and $K\left(s, s^{\prime}\right)$ are both continuous for $a \leq s \leq b, a \leq s^{\prime} \leq b$ then, by the Fredholm theory, condition (2) implies (1), since, as we showed earlier in the present section, (2) assures the non-existence of a characteristic solution. 


$$
\left|\eta_{n}\right| \leq \frac{(a-b) p^{n}}{2}
$$

However, from the practical (computational) point of view such estimates are of little or no use in determining the accuracy that has been attained at any stage of the procedure. For example, in one of the numerical illustrations to be presented in Sec. 8, $a=1, b=-1$, and $p=.75$, so that if $f_{0}$ is chosen $\equiv 0$, the upper bound $M_{0}=1$ is obtained $^{\mathbf{1 0}}$ for $\left|\eta_{0}\right|$, and therefore, in order to be certain that the error is everywhere less than .01 in absolute value, it would appear necessary, according to (3.11), to perform about 16 successive approximations, ${ }^{11}$ since $(.75)^{16} \approx .01$. It is necessary to have a more delicate criterion than (3.11) for estimating the accuracy of the approximations, and also to have a method for determining a suitable initial approximation $f_{0}$. In this section a better estimate than that given by (3.11) is obtained (Theorem 4.1), as well as two theorems of lesser importance, and in Sec. 7 the choice of a suitable $f_{0}$ will be discussed.

Theorem 4.1. ${ }^{12}$ If $\max \left|f_{n}-f_{n+1}\right|=A$, then $M_{n}=\max \left|\eta_{n}\right| \leq A /(1-p)$.

Proof: Since $f_{n}-f_{n+1}=\eta_{n}-\eta_{n+1}$, the hypothesis may be written as follows:

$$
\left|\eta_{n}-\eta_{n+1}\right| \leq A \text {. }
$$

Adding $\left|\eta_{n+1}\right|$ to both sides of (4.1), and recalling the triangle inequality, one obtains

$$
\left|\eta_{n}\right| \leq A+\left|\eta_{n+1}\right| \text {. }
$$

Since $\left|\eta_{n+1}\right| \leq p M_{n},(4.2)$ yields

$$
\left|\eta_{n}\right| \leq A+p M_{n} \text {. }
$$

Since the right-hand side of (4.3) is independent of the point at which $\eta_{n}$ is evaluated, $\left|\eta_{n}\right|$ can be replaced by $M_{n}$, so that one obtains

$$
M_{n}(1-p) \leq A .
$$

Dividing both sides of (4.4) by the positive quantity $1-p$, we obtain the statement of the theorem.

Theorem 4.2. If $\max \left|f_{n}-f_{n+1}\right|=A$, then $M_{n}=\max \left|\eta_{n}\right| \geq A /(1+p)$.

Proof. Given any $\epsilon>0$, there can be found a value of $s$, say $s=s_{0}$, for which

$$
\left|f_{n}\left(s_{0}\right)-f_{n+1}\left(s_{0}\right)\right|=\left|\eta_{n}\left(s_{0}\right)-\eta_{n+1}\left(s_{0}\right)\right| \geq A-\epsilon .
$$

Now, since $\left|\eta_{n}\left(s_{0}\right)-\eta_{n+1}\left(s_{0}\right)\right| \leq\left|\eta_{n}\left(s_{0}\right)\right|+\left|\eta_{n+1}\left(s_{0}\right)\right|$, one obtains from (4.5) the further inequality

$$
\left|\eta_{n}\left(s_{0}\right)\right|+\left|\eta_{n+1}\left(s_{0}\right)\right| \geq A-\epsilon .
$$

But $\left|\eta_{n}\left(s_{0}\right)\right| \leq M_{n},\left|\eta_{n+1}\left(s_{0}\right)\right| \leq M_{n+1} \leq p M_{n}$. Therefore,

${ }^{10}$ If any other choice of $f_{0}$ is made, the best upper bound that can be given for $M_{0}$ entirely on the basis of the principle of maximum and minimum will exceed 1.

"Here the additional difficulty is overlooked that at each stage of an actual computation it will almost certainly be necessary to approximate $f_{n}$ by a simpler function in order to evaluate the succeeding function $f_{n+1}$, thus increasing the best bound that can be given for the maximum error.

'In Theorems 4.1 and 4.2 the term "max" stands for "least upper bound", as in Sec. 3. 


$$
M_{n}(1+p) \geq A-\epsilon .
$$

On account of the arbitrariness of the positive number $\epsilon,(4.7)$ implies that

$$
M_{n}(1+p) \geq A \text {. }
$$

Dividing both sides of (4.8) by $1+p$, we obtain the statement of Theorem 4.2.

Theorem 4.3. If $f_{n} \leq f_{n+1}$ everywhere, then for $m>n, f_{m} \leq f_{m+1} \leq f$.

Proof. The hypothesis may be written in the form

$$
\eta_{n}-\eta_{n+1} \leq 0 .
$$

From (3.9) it is apparent that

$$
\eta_{n+1}-\eta_{n+2}=\int\left(\eta_{n}-\eta_{n+1}\right) K_{R} d s^{\prime} .
$$

Since $K_{R}$ is everywhere non-negative, it is clear that $\eta_{n+1}-\eta_{n+2} \leq 0$, and hence $f_{n+1} \leq$ $f_{n+2}$. By repeating this argument, one obtains $f_{m} \leq f_{m+1}$ for $m \geq n$. Since $f_{m} \rightarrow f$, it. follows that each estimate $f_{m}$ lies below $f$.

This theorem obviously holds also with the inequalities reversed. Therefore, if two initial estimates $f_{0}^{(1)}$ and $f_{0}^{(2)}$ are chosen such that $f_{0}^{(1)} \leq f_{1}^{(1)}$ and $f_{0}^{(2)} \geq f_{1}^{(2)}$, the solution $f$ of (2.6) is "squeezed" between the terms of the sequences $\left\{f_{n}^{(1)}\right\}$ and $\left\{f_{n}^{(2)}\right\}$.

5. Discussion of the inequality (3.3). In Sec. 3 a procedure was presented for the solution of the integral equation (2.6). The discussion was incomplete in one respect, for it was necessary to assume that the function $R(s)$ can be so chosen that the inequality (3.3) is satisfied. In Sec. 8 it will be shown, by an explicit computation, that (3.3) can be satisfied in the particular case of a domain bounded by any finite number of collinear slits. It appears, however, that in order to extend this result to more general types of domains it is necessary to impose certain restrictions upon the given domain and on the added arcs. Rather than attempting to state very general conditions, we shall here consider a rather restricted type of domain, but it will be apparent from the argument how one might proceed in the investigation of other domains.

It will be recalled from Sec. 2 that the integral $\int K_{R}\left(s, s^{\prime}\right) d s^{\prime}$, extended over the added arcs, is simply the mean value over the circumference of the circle $C_{R}(P$ ) (where $P$ is the inner point of the added arcs corresponding to the parameter $s$ ) of the harmonic measure of the added arcs. ${ }^{13}$ If the function $R(s)$ is continuous on each of the added arcs, as we shall assume, then the above integral is also a continuous function of $s$, and is less than unity, for the harmonic measure of the added arcs is never actually equal to unity (except possibly at the finite number of points where the added arcs meet the circumference). Therefore, in any particular case it is only necessary to show that the mean value is bounded away from unity in some neighborhood of each endpoint of the added arcs.

We shall now assume that the original (bounded) domain $S$ and the added arcs. $C D$ and $E F$, as shown in Fig. 2, are such that the tangent to the added arc at each point $\mathrm{P}$ has no points in common with one of the two subdomains ( $S_{2}$ in the figure) and meets

${ }^{13}$ The harmonic measure is taken, we recall, with respect to the subdomain in which the variable point on the circumference lies, not with respect to the original domain. 
the boundary of the other subdomain $\left(S_{1}\right)$ only at two points $A$ and $B$ (in addition to the point of tangency $P$ ). The points $A$ and $B$ will vary, of course, with the point $P$. Now, the mean value of the harmonic measure of the added $\operatorname{arcs} C D$ and $E F$ will surely be bounded below unity as $P$ varies along these arcs if the mean value on the semicircle lying in the region ${ }^{14} S_{P}$ bounded by the segment $A P B$ and the arc $A G B$ is bounded

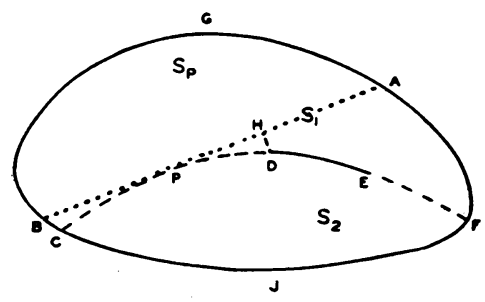

FIG. 2.

below unity, for the least upper bound of the mean value on the other semi-circle cannot exceed unity. Also, the harmonic measure of the added arcs at any point of the former semi-circle $^{15}$ is less than that of the curve $B C D E F A$, which, in turn, by the so-called "principle of extension" [3, p. 63], is less than that of the line segment $A B$ with respect to the domain $S_{P}$; the latter harmonic measure, furthermore, is less than that of the segment $A B$ with respect to the entire half-plane (containing $S_{P}$ ) determined by extending $A B$ to infinity in both directions. Now, we make the additional assumption that, as $P \rightarrow C, R$ can be chosen $=c \overline{P B}$, where $c$ is a sufficiently small positive constant. (This would not be possible, for example, if the arcs $C D$ and $C J F$ meet at $C$ under zero angle.) Then, as is shown in Sec. 8, the mean value on the semi-circle of the harmonic measure of $A B$ with respect to the half-plane will be bounded below unity. ${ }^{16}$ Therefore, the inequality (3.3) holds as $P \rightarrow C$.

We now turn to the case when $P \rightarrow D$. Since the distance (in the usual point-set sense) between $C_{R}(P)$ and the arc $E F$ is bounded away from zero, we may disregard the harmonic measure of this arc, and consider only the mean value over the semicircle lying in $S_{P}$ of the harmonic measure of the arc $C D$ (with respect to $S_{1}$ ). We now assume that the arc $C D$ is continuously curved in a neighborhood of $D$, so that the length of the segment $D H$ of the perpendicular to $A B$ from $D$ satisfies, for some sufficiently large positive number $\sigma$ and for $P$ sufficiently close to $D$, the inequality

$$
\overline{D H} \leq \sigma \overline{P H}^{2} .
$$

We assume further that the point $P$ and the entire arc $D E F$ lie on opposite sides of the line determined by $D$ and $H$. Then, by the aforementioned "principle of extension", the mean value over the semi-circle lying in $S_{P}$ of the harmonic measure of the arc $C D$ with respect to $S_{1}$ is less than the mean value of the harmonic measure of the broken line segment $B H D$ with respect to the three-quarter-plane determined by extending

${ }^{14}$ The subscript $P$ serves as a reminder that this domain depends upon the point $P$.

${ }^{15}$ For this semi-circle the harmonic measure will always be taken with respect to $S_{1}$.

${ }^{16}$ The fact that the segment $A B$ changes direction as $P$ varies obviously causes no difficulty. The change in length of $A B$ can be eliminated from our considerations by considering the harmonic measure of a (finite) segment, beginning at $B$, whose length exceeds the maximum length of $A B$. The considerations of Sec. 8 are then immediately applicable. 
$H B$ and $H D$ beyond $B$ and $D$ respectively to infinity. See Fig. 3. This mean value, in turn, is majorized by the mean value of the harmonic measure (still with respect to the three-quarter-plane) of the entire half-line $H B \infty$ plus that of $H J$, where $\overline{H J}$ is equal

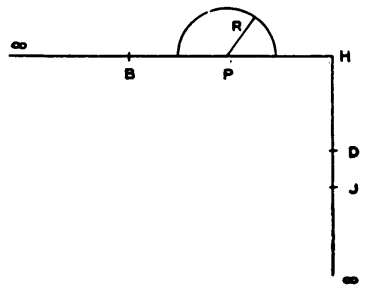

FIG. 3.

to the right-hand side of $(5.1) .{ }^{17}$ (As pointed out previously, we may assume $\overline{P H}$ so small that (5.1) holds.) We now assume that $R$ is chosen as follows:

$$
R=\gamma \overline{P H},
$$

where $\gamma$ is some positive constant $<1$. Then, from similarity considerations, it is apparent that the mean value of the harmonic measure of $H B \infty$ remains constant (and therefore certainly $<1$ ) as $P$ approaches $D$. It also follows from similarity considerations, together with (5.1) and (5.2), that the contribution from $H J$, and therefore that from $H D$, diminishes to zero as $P$ approaches $D$. Therefore the mean value of the harmonic measure of $D H B$ is certainly bounded away from unity. From the chain of inequalities which we have established it follows a fortiori that $\int K_{R}\left(s, s^{\prime}\right) d s^{\prime}$ is bounded away from unity as $P$ approaches $D$.

Finally, we note that the discussions of the cases $P \rightarrow E$ and $P \rightarrow F$ are entirely parallel to those for $P \rightarrow D$ and $P \rightarrow C$ respectively.

While the discussion presented here deals with a rather specialized type of domain, it seems likely that attempts to prove that the inequality (3.3) can be satisfied for a given domain must in almost all cases proceed along the lines used here-i.e., of repeated use of the "principle of extension"; for in only very few cases (of which that considered in Secs. 6 and 8 is one) is it possible to evaluate the integral $\int K_{R}\left(s, s^{\prime}\right) d s^{\prime}$ in closed form.

6. Specific application to "slit case". In this section the method described in the first part of this paper will be applied to a rather special type of domain which is, however, of considerable interest.

Let the domain $S$ for which the Dirichlet problem is to be solved consist of the entire $(x, y)$-plane except for a finite number, $n$, of collinear slits, which constitute the boundary of $S$. Without loss of generality (since the Laplace equation is invariant under rotation and translation) these slits may be considered to lie on the $x$-axis. Also it may be assumed that the "point at infinity" is an inner ${ }^{18}$ boundary point of $S$, for this can always be realized by performing an inversion in a circle with center at any inner point of any one of the slits. (The two-dimensional Laplace equation is invariant under inversion

\footnotetext{
17The orientation of the boundary of the new domain will vary with $P$, but this does not cause any difficulty.

${ }^{18}$ That is, the slits extend to infinity both on the right and on the left.
} 
as well as rotation and translation.) The portion of the $x$-axis consisting of the slits will henceforth be designated as $C$ and the remainder as $D$ (which consists of the $n$ intervals $\left.\left(a_{1}, b_{1}\right),\left(a_{2}, b_{2}\right), \cdots,\left(a_{n}, b_{n}\right)\right)$. There is now defined on $C$ a function ${ }^{19} h(x)$, which is assumed to be bounded on each slit and continuous except perhaps at a finite number of points. The theorem referred to in Sec. 2 from the Nevanlinna treatise is directly applicable, so that we are assured of the existence of a unique function $u(P)$, $P=(x, y)$, which is harmonic and bounded in $S$ and approaches $h(x)$ whenever $P$ approaches a point of $C$ at which $h(x)$ is continuous.

Now, it is clear that the problem would be essentially solved if the values of $u(P)$ could be determined along each of the intervals $\left(a_{1}, b_{1}\right),\left(a_{2}, b_{2}\right), \cdots,\left(a_{n}, b_{n}\right)$ constituting $D$, for then the values of $u(P)$ could be determined at all points not on the $x$-axis by the Poisson integral formula:

$$
u(P)=\frac{|y|}{\pi} \int_{-\infty}^{\infty} \frac{u(\xi, 0) d \xi}{(\xi-x)^{2}+y^{2}}, \quad(y \neq 0) .
$$

Letting $u(x, 0)$ on $D$ be designated as the function $f(x)$, we may rewrite (6.1) in the form

$$
u(P)=\frac{|y|}{\pi} \int_{C} \frac{h(\xi) d \xi}{(\xi-x)^{2}+y^{2}}+\frac{|y|}{\pi} \int_{D} \frac{f(\xi) d \xi}{(\xi-x)^{2}+y^{2}}, \quad(y \neq 0) .
$$

Let $(x, 0)$ be any point, temporarily fixed, interior to $D$, say inside the interval $\left(a_{k}, b_{k}\right)$ and let $R$ be any positive number subject only to the condition

$$
R \leq \min \left(x-a_{k}, b_{k}-x\right) ;
$$

then the mean-value property for $u(P)$ at the point $(x, 0)$ may be expressed (on account of the evenness of $u(P)$ in $y$, which is apparent from (6.1)) as follows:

$$
f(x)=\frac{1}{\pi} \int_{0}^{\pi} u(x+R \cos \theta, R \sin \theta) d \theta .
$$

Now, for $0<\theta<\pi$, equation (6.2) may be written in the form

$$
\begin{aligned}
u(x+R \cos \theta, R \sin \theta) & =\frac{R \sin \theta}{\pi} \int_{C} \frac{h(\xi) d \xi}{(\xi-x-R \cos \theta)^{2}+R^{2} \sin ^{2} \theta} \\
& +\frac{R \sin \theta}{\pi} \int_{D} \frac{f(\xi) d \xi}{(\xi-x-R \cos \theta)^{2}+R^{2} \sin ^{2} \theta} .
\end{aligned}
$$

We now proceed as explained in Sec. 2. The integrand of (6.4) is replaced by the right-hand side of (6.5), the order of integration is inverted, and the inner integration. is performed. In this manner, we obtain

${ }^{19}$ More generally, different functions could be prescribed on the upper and lower edges of $C$. (In the discussion of the more general case in Sec. 2 this possibility was considered.) If different boundary values $h^{+}(x)$ and $h^{-}(x)$ were prescribed on the upper and lower edges of $C$ respectively, the problem could easily be reduced to one of the type considered here by dividing it into two problems, one with boundary values $\frac{1}{2}\left(h^{+}(x)+h^{-}(x)\right)$ on both edges of $C$, and the other with boundary values $\frac{1}{2}\left(h^{+}(x)-h^{-}(x)\right)$ and $\frac{1}{2}\left(h^{-}(x)-\right.$ $h^{+}(x)$ ) on the upper and lower edges respectively. For the second problem the solution must obviously vanish on $D$ (by symmetry considerations), so that by using the Poisson formula for the upper and lower half-planes in turn the second problem would be solved, leaving only a problem of the kind considered here. 


$$
f(x)=\int_{C} h(\xi) K(x, R, \xi) d \xi+\int_{D} f(\xi) K(x, R, \xi) d \xi,
$$

where

$$
K(x, R, \xi)=\left(\pi^{2}(\xi-x)\right)^{-1} \log \left|\frac{\xi-x+R}{\xi-x-R}\right| .
$$

(When $\xi=x, K(x, R, \xi)$ is to be taken equal to $2 / \pi^{2} R$.)

Now, for each value of $x$ interior to $D$ let a positive $R$ be chosen, subject only to the restriction (6.3); i.e., a function $R(x)$ is chosen. Then the first term on the righthand side of (6.6) becomes a definite function of $x$, defined in the interior of $D$. This function will be designated $g_{R}(x)$, the subscript $R$ indicating, as in Sec. 2, that this function depends on the choice made for the function $R(x)$. For $\xi$ interior to $D$, the righthand side of (6.7) becomes a definite function of $\xi$ and $x$, with $x$ also assuming values in the interior of $D$. This function will be designated $K_{R}(x, \xi)$. Thus, there is obtained for $f(x)$ an integral equation (or, in a sense, a family of integral equations, one for each possible choice of the function $R(x)$ ):

$$
f(x)=g_{R}(x)+\int_{D} K_{R}(x, \xi) f(\xi) d \xi .
$$

In Sec. 8 we shall first show that the function $R(x)$ can be so chosen that condition (3.3) is satisfied, so that the method prescribed in Sec. 3 for the solution of (6.8) (or, more generally (2.6)) is applicable, and then shall illustrate our method by means of two numerical examples. Before this, however, we shall present in Sec. 7 a brief discussion of the problem of choosing a suitable approximation with which to begin the procedure described in Sec. 3.

7. Choice of an initial approximation. Although the sequence of functions $\left\{f_{n}(s)\right\}$ obtained by the procedure described in Sec. 3 converges to $f(s)$, the solution of the integral equation (2.6), regardless of whether $f_{0}(s)$ is a good or poor approximation to $f(s)$, it is essential from the practical viewpoint to be able to select a suitable initial approximation, for with a poor choice of $f_{0}(s)$ the number of succeeding functions $f_{1}(s), f_{2}(s), \cdots$ which would be necessary to provide a sufficiently accurate approximation to $f(s)$ might be so large as to make our procedure useless for computational purposes. For simplicity we shall restrict ourselves to the consideration of domains of the type considered in Sec. 6, and, correspondingly, to the problem of solving the integral Eq. (6.8), whose kernel is defined by (6.7); it will be apparent, however, that the basic idea which we shall employ is also applicable in the general case.

It is obvious that Eq. (6.8) may be expressed in variational terms as the problem of finding a function $f(x)$ for which

$$
\int_{D}\left(f(x)-g_{R}(x)-\int_{D} f(\xi) K_{R}(x, \xi) d \xi\right)^{2} d x=\text { minimum, }
$$

where the class of admissible functions may be taken as that of all functions which are bounded and continuous in the interior of each interval of $D$. Now, following the method of Rayleigh-Ritz [1, pp. 149-151], we would replace $f(x)$ in (7.1) by a function $F(x, \alpha, \beta, \gamma, \cdots)$ of $x$ and a number of parameters $\alpha, \beta, \gamma, \cdots$ (and correspondingly replace $f(\xi)$ by $F(\xi, \alpha, \beta, \gamma, \cdots))$, and then choose the parameters so as to minimize 
the left-hand side of (7.1). The function obtained with this choice of parameters is then the best approximate solution to (7.1) among the class of functions considered, and may therefore reasonably be expected to serve as a good initial approximation for the procedure described in Sec. 3. Actually, from Theorems 4.1 and 4.2 it is seen that it would be preferable to select the parameters so as to minimize

$$
\max _{x \varepsilon D}\left|F(x, \alpha, \beta, \gamma, \cdots)-g_{R}(x)-\int_{D} F(\xi, \alpha, \beta, \gamma, \cdots) K_{R}(x, \xi) d \xi\right|,
$$

but it is evident that this is in general a hopelessly difficult task. Whether or not the initial approximation obtained by minimizing the left-hand side of (7.1) is actually a good one can then be seen by evaluating the expression (7.2).

In particular, we may take $F(x, \alpha, \beta, \gamma, \cdots)$ to be a polynomial in $x$ whose coefficients are the parameters $\alpha, \beta, \gamma, \cdots$ (cf. Example 1, Sec. 8) or, more generally, $F(x, \alpha, \beta, \gamma, \cdots)$ may consist of different polynomials in the different intervals of $D$ (cf. Example 2, Sec. 8). As may be seen by performing the substitution

$$
\xi=x+R u,
$$

the integral

$$
\int_{D} F(\xi, \alpha, \beta, \gamma, \cdots) K_{R}(x, \xi) d \xi
$$

can be expressed as a sum of integrals of elementary type plus a single non-elementary integral, namely

$$
\int u^{-1} \log \left|\frac{1+u}{1-u}\right| d u
$$

which can be easily evaluated by series methods. However, even when $F(x, \alpha, \beta, \gamma, \cdots)$ is of the simple form considered here, the minimizing of the left-hand side of (7.1) is not a simple task, for the integration with respect to $x$ involves non-elementary functions. Nevertheless, the best values of the parameters $\alpha, \beta, \gamma, \cdots$ may be determined at least approximately by replacing the integration with respect to $x$ by a summation, say by using Simpson's rule. This is the method which was employed in Sec. 8. In Example 1, the interval $-1 \leq x \leq 1$ was divided into 20 equal subintervals, and in Example 2 each of the intervals $-4 \leq x \leq-1$ and $1 \leq x \leq 4$ was divided into 12 equal sub-intervals.

The fact that very satisfactory initial approximations were obtained in Sec. 8 by the method which we have described here suggests that the variational approach is a suitable means for obtaining an initial approximation, and also that the approximate method described in the above paragraph for obtaining the best values of the parameters yields values not differing appreciably from the correct ones.

8. Illustrative examples for domains bounded by collinear slits. In proving the convergence of the approximation procedure in Sec. 3 we had to assume that the inequality (3.3) is satisfied. In this section we shall first of all show that, for the domains considered in Sec. 6 , it is indeed possible to assure, by a suitable choice of the function $R(x)$, that (3.3) holds.

The function $R(x)$ will be chosen as large as possible. Therefore,

$$
R(x)=\min \left(x-a_{k}, b_{k}-x\right),
$$


where $x$ lies in the interior of the interval $\left(a_{k}, b_{k}\right)$. In order to show that with this choice of $R(x)$ a constant $p$ exists for which (3.3) holds, we consider the function

$$
p(x)=\int_{D} K_{R}(x, \xi) d \xi=\frac{1}{\pi^{2}} \int_{D}(\xi-x)^{-1} \log \left|\frac{\xi-x+R}{\xi-x-R}\right| d \xi,
$$

defined for all values of $x$ interior to $D$. If $x$ lies in the left half of the $k^{\prime}$ th interval, $\left(a_{k}, b_{k}\right)$, so that $R=x-a_{k}$, we may write (8.2) in the form:

$$
p(x)=\frac{1}{\pi^{2}} \int_{D}(\xi-x)^{-1} \log \left|\frac{\xi-a_{k}}{\xi-2 x+a_{k}}\right| d \xi .
$$

Since the integrand is positive everywhere, the right-hand side of (8.3) is increased if the integration is extended over a point set containing $D$. Extending the integration over all values of $\xi$ except for the segment of $C$ immediately to the left of the $k^{\prime}$ th interval $^{20}$ of $D$, we therefore obtain the inequality

$$
\begin{aligned}
p(x)<\frac{1}{\pi^{2}} \int_{-\infty}^{\infty}(\xi-x)^{-1} \log \left|\frac{\xi-a_{k}}{\xi-2 x+a_{k}}\right| d \xi \\
\quad-\frac{1}{\pi^{2}} \int_{b_{k-1}}^{a_{k}}(\xi-x)^{-1} \log \left|\frac{\xi-a_{k}}{\xi-2 x+a_{k}}\right| d \xi .
\end{aligned}
$$

Performing in (8.4) the substitution

$$
\xi=x+R u=x+\left(x-a_{k}\right) u,
$$

we obtain

$$
p(x)<\frac{1}{\pi^{2}} \int_{-\infty}^{\infty} u^{-1} \log \left|\frac{1+u}{1-u}\right| d u-\frac{1}{\pi^{2}} \int_{\left(b_{k-1}-x\right) /\left(x-a_{k}\right)}^{-1} u^{-1} \log \left|\frac{1+u}{1-u}\right| d u .
$$

Now, it is known ${ }^{21}$ that

$$
\frac{1}{\pi^{2}} \int_{-\infty}^{\infty} u^{-1} \log \left|\frac{1+u}{1-u}\right| d u=1 .
$$

Also, since the integrand of the second term on the right-hand side of (8.6) is always positive, the entire right-hand side is largest when the lower limit of integration is as large (i.e., as small in absolute value) as possible. This clearly occurs when $x$ is as large as possible, i.e., when $x=1 / 2\left(a_{k}+b_{k}\right)$, so that, for $a_{k}<x \leq 1 / 2\left(a_{k}+b_{k}\right)$, the following inequality holds:

$$
p(x) \leq 1-\frac{1}{\pi^{2}} \int_{\left[2 b_{k-1}-\left(a_{k}+b_{k}\right)\right] /\left(b_{k}-a_{k}\right)}^{-1} u^{-1} \log \left|\frac{1+u}{1-u}\right| d u .
$$

${ }^{20}$ If $k=1$, the interval $\left(-\infty, a_{1}\right)$ is to be excluded from the integration. In (8.4), $b_{0}$ is therefore to be taken as $-\infty$.

${ }^{21}$ This can be seen without performing any computations, in the following way. By taking account of the substitution (8.5), one sees that the left-hand side of (8.7) is equal to the first term on the right-hand side of (8.4). The latter quantity, in turn, is, by the considerations of Sec. 6, equal to the mean value over a certain semi-circle of the harmonic measure of the entire $x$-axis with respect to the half-plane $y=0$. Since the harmonic measure of the entire boundary of any domain is identically equal to one at all points of the domain, (8.7) follows. 
Similarly, it is possible to obtain for $p(x)$ an upper bound less than one in the right half of each interval of $D$. If $p$ is taken equal to the largest of the $2 n$ upper bounds so obtained, the inequality (3.3) will be satisfied.

A similar proof can be carried out for various other choices of $R(x)$; e.g., one may choose $R(x)=c \min \left(x-a_{k}, b_{k}-x\right)$, where $c$ is any positive constant $<1$. However, the value of $p$ obtained with any $c<1$ would be closer to one than with $c=1$, so that the choice $c=1$ appears best. In the numerical examples to be presented here $R(x)$ is always chosen with $c=1$, i.e., according to (8.1). In Example 1, we have $p=.75$; in Example 2, $p=.8387$.

Example 1. The domain $S$ consists of the entire $(x, y)$-plane cut by the slits $(-\infty,-1)$ and $(1, \infty)$ of the $x$-axis, on which the boundary values $h(x)=-1$ and $h(x)=1$ respectively are prescribed.

The exact solution is easily found to be given, on the interval $(-1,1)$ of the $x$-axis, by

$$
u(x, 0)=f(x)=\frac{2}{\pi} \arcsin x ;
$$

however, in order to illustrate our procedure, we have taken three different initial approximations, which we designate $f_{0}^{(1)}(x), f_{0}^{(2)}(x), f_{0}^{(3)}(x)$, and have determined the corresponding succeeding approximations, which we designate $f_{1}^{(1)}(x), f_{1}^{(2)}(x), f_{1}^{(3)}(x)$, respectively. The results of these computations are presented in Table 1. (Only positive values of $x$ are listed, since all functions appearing are odd.)

TABLE 1

\begin{tabular}{cccccccc}
\hline \hline$x$ & $f_{0}(x)$ & $f_{1}(x)$ & $f_{0}(x)$ & $f_{1}(x)$ & $f_{0}(x)$ & $f_{1}(x)$ & $f(x)$ \\
\hline 0 & 0 & 0 & 0 & 0 & 0 & 0 & 0 \\
.1 & .1 & .0589 & .0600 & .0624 & .0600 & .0652 & .0638 \\
.2 & .2 & .1268 & .1215 & .1281 & .1215 & .1317 & .1282 \\
.3 & .3 & .2042 & .1860 & .1935 & .1860 & .1979 & .1940 \\
.4 & .4 & .2894 & .2550 & .2613 & .2550 & .2665 & .2620 \\
.5 & .5 & .3819 & .3300 & .3317 & .3300 & .3375 & .3333 \\
.6 & .6 & .4820 & .4126 & .4069 & .4126 & .4113 & .4097 \\
.7 & .7 & .5920 & .5041 & .4907 & .5041 & .4977 & .4936 \\
.8 & .8 & .7072 & .6062 & .5885 & .6062 & .5976 & .5903 \\
.9 & .9 & .8388 & .7203 & .7098 & .7203 & .7250 & .7129 \\
1.0 & 1. & 1. & .8479 & .8859 & 1. & 1. & 1. \\
\hline
\end{tabular}

The first initial approximation to be chosen was:

$$
f_{0}^{(1)}(x)=x .
$$

This function, although joining continuously with the prescribed boundary values, is clearly a poor choice; nevertheless, as may be seen from Table 1, the succeeding approximation, $f_{1}^{(1)}(x)$, shows a very marked tendency toward the exact solution given by (8.9).

The function $f_{0}^{(2)}(x)$ was chosen to be the "best" third-degree polynomial (in the sense of Sec. 7). We thus obtained: 


$$
f_{0}^{(2)}(x)=.5974 x+.2505 x^{3} .
$$

This function is seen to be a rather good approximation to the exact solution except near the end-points of the range, for it fails to join continuously with the prescribed boundary-values. The succeeding approximation $f_{1}^{(2)}(x)$ is seen to be still closer to $f(x)$ for most values of $x$, and the improvement near the ends is worthy of note.

The third choice of an initial approximation was made as follows: The function (8.11) was retained in the sub-interval $-.9 \leq x \leq .9$, but for $-1 \leq x \leq-.9$ and for $.9 \leq x \leq 1$ was replaced by second-degree polynomials joining smoothly with (8.11) at $x= \pm .9$ and continuously with the prescribed boundary values at $x= \pm 1$. In this way the following choice was obtained for $f_{0}^{(3)}(x)$ :

(a) $f_{0}^{(3)}(x)=-12.5230-27.4344 x-15.9114 x^{2}, \quad-1 \leq x \leq-.9$;

(b) $f_{0}^{(3)}(x)=f_{0}^{(2)}(x)=.5974 x+.2505 x^{3}, \quad-.9 \leq x \leq .9$;

(c) $f_{0}^{(3)}(x)=12.5230-27.4344 x+15.9114 x^{2}, \quad .9 \leq x \leq 1$.

The corresponding approximation $f_{1}^{(3)}(x)$ is seen to provide a remarkably good approximation to $f(x)$ throughout the range $(-1,1)$.

Example 2. The domain $S$ consists of the entire $(x, y)$-plane cut by the slits $(-\infty,-4),(-1,1)$, and $(4, \infty)$ of the $x$-axis, on which the boundary values $h(x)=$ $0,1,0$ respectively are prescribed..$^{22}$

The exact solution to this problem can be expressed in terms of elliptic integrals. In particular, on the interval $(1,4)$ of the $x$-axis, the solution is given by

$$
u(x, 0)=f(x) \doteq \frac{1}{2}-\frac{\int_{0}^{w}\left(1-t^{2} / 81\right)^{-\frac{1}{2}}\left(1-t^{2}\right)^{-\frac{1}{2}} d t}{2 \int_{0}^{1}\left(1-t^{2} / 81\right)^{-\frac{1}{2}}\left(1-t^{2}\right)^{-\frac{1}{2}} d t}
$$

where

$$
w=\frac{3(x-2)}{x+1} .
$$

In the notation commonly used for elliptic integrals, (8.13) may be written in the form

$$
f(x)=\frac{1}{2}-\frac{K(\arcsin w, 1 / 9)}{2 K(1 / 9)} .
$$

(Since the solution is obviously even in $x$, it suffices to determine $f(x)$ in the interval $(1,4)$.)

As an initial approximation we first tried

$$
f_{0}^{(1)}(x)=\frac{1}{3}(4-|x|),
$$

which is seen to join continuously with the prescribed boundary values. (As in Example 1, a superscript is attached to the approximation function in order to distinguish it from another one which will be used later.) As in the case of the first initial approximation considered in Example 1, this is a rather poor choice; nevertheless, as may be seen from

${ }^{22}$ That is, the problem is that of determining the harmonic measure of the finite slit. 
Table 2, the succeeding approximation $f_{1}^{(1)}(x)$ shows a strong tendency toward the exact solution $f(x)$, whose values are also given in Table 2 .

TABLE 2

\begin{tabular}{lccccc}
\hline \hline \multicolumn{1}{c}{$x$} & $f_{0}(x)$ & $f_{1}(x)$ & $f_{0}(x)$ & $f_{1}(x)$ & $f(x)$ \\
\hline $1+0$ & 1. & 1. & .8985 & .9239 & 1. \\
1.25 & .9167 & .8549 & .7629 & .7471 & .7430 \\
1.5 & .8333 & .7499 & .6537 & .6419 & .6402 \\
1.75 & .7500 & .6535 & .5664 & .5648 & .5639 \\
2.0 & .6667 & .5687 & .4966 & .5014 & .5000 \\
2.25 & .5833 & .4956 & .4397 & .4443 & .4438 \\
2.5 & .5000 & .4366 & .3913 & .3925 & .3921 \\
2.75 & .4167 & .3895 & .3470 & .3442 & .3434 \\
3.0 & .3333 & .3461 & .3023 & .3002 & .2956 \\
3.25 & .2500 & .2819 & .2527 & .2468 & .2472 \\
3.50 & .1667 & .2062 & .1937 & .1953 & .1954 \\
3.75 & .0833 & .1168 & .1209 & .1309 & .1342 \\
$4-0$ & 0 & 0 & .0300 & .0225 & 0 \\
\hline
\end{tabular}

As in Example 1, we next obtained another approximation by the method described in Sec. 7. The approximation was taken to be of the form

$$
\begin{array}{ll}
f_{0}^{(2)}(x)=a+b x+c x^{2}+d x^{3}, & 1 \leq x \leq 4 ; \\
f_{0}^{(2)}(x)=a-b x+c x^{2}-d x^{3}, & -4 \leq x \leq-1,
\end{array}
$$

the coefficients $a, b, c, d$ being unspecified to begin with. (From (8.17) it is apparent that $f_{0}^{(2)}(x)$ is even, so that as before it suffices to determine $f_{1}^{(2)}(x)$ for $1 \leq x \leq 4$.) In this manner the following values were found for the coefficients:

$$
a=1.7936, \quad b=-1.2369, \quad c=.3894, \quad d=-.0476 \text {. }
$$

With this choice of the coefficients, the values of $f_{0}^{(2)}(x)$ and $f_{1}^{(2)}(x)$ listed in Table 2 were obtained. As in the eorresponding part of Example 1, these functions are seen to be rather good approximations to the exact solution, except near the end-points. It is almost certain that if $f_{0}^{(2)}(x)$ were modified near the end-points so as to join continuously with the prescribed boundary-values, as was done in Example 1, both the initial and succeeding approximations would constitute considerable improvements over the ones which we have obtained.

\section{BIBLIOGRAPHY}

1. Couranit-Hilbert, Methoden der mathematischen Physik, Vol. I, 2nd ed., 1931.

2. Courant-Hilbert, Methoden der mathematischen Physik, Vol. II, 1937.

3. R. Nevanlinna, Eindeutige analytische Funktionen, 1936.

4. R. Nevanlinna, Crelles Journal 180, 121-128 (1938).

5. S. Saks, Theory of the integral, 1937. 